\title{
Comparison of Polyphenol Content and Antioxidant Capacity of Colored Potato Tubers, Pomegranate and Blueberries
}

Diganta Kalita and Sastry S Jayanty*

Department of Horticulture and Landscape Architecture, San Luis Valley Research Center, Colorado State University, 0249 East County, Road 9N Center, CO 81125, USA

\begin{abstract}
Dietary antioxidants from fruits and vegetables play an important role in preventing free radical-induced oxidative damage to lipids, proteins, and nucleic acids. Potato tubers with purple- and red-colored flesh contain high levels of phenolics and anthocyanins, as do pomegranates and blueberries. The present study evaluates the antioxidant potential of colored-flesh potato tubers in comparison with polyphenol-rich fruits, blueberries and pomegranate juice. The ranges of total phenolics (TP), total anthocyanins (TA), and total flavonoids (TF) were $(4482-11189 \mu \mathrm{g} / \mathrm{g}),(1889-6289 \mu \mathrm{g} / \mathrm{g})$, and $(140-503 \mu \mathrm{g} / \mathrm{g})$, respectively. The potential antioxidant capacities of the potato tubers were similar to that of pomegranate and blueberry. High correlations of TP, TA, and TF with antioxidant activity, such as ORAC, ABTS, and DPPH ( $r=0.95,0.93$, and 0.80 for TP; $r=0.93,0.96$, and 0.54 for TA; $r=0.80,0.91$, and 0.70 for TF), were found for the materials tested. The total antioxidant activity values per serving size of baked red and purple potatoes, blueberries, and pomegranate juice were also found to have comparable ranges.
\end{abstract}

Keywords: Polyphenols; Antioxidant activity; Total antioxidant capacity; Red/purple potato; Pomegranate; Blueberries

\section{Introduction}

Polyphenolic compounds are secondary plant metabolites and constitute the largest group of health-promoting phytochemicals [12]. These compounds are known to possess significant antioxidant, antiglycemic, antiviral, anticarcinogenic, and anti-inflammatory activities and exhibit anti-allergic and antimicrobial properties [3-5]. Antioxidants, which have been defined by the Institute of Medicine of the National Academy of Sciences, USA, as "a substance in foods that significantly decreases the adverse effects of reactive species on normal physiological function in humans", can also prevent the oxidative damage of lipids, proteins, and nucleic acids caused by superoxide, hydroxyl, peroxyl, and alkoxyl free radicals. Antioxidants play a critical role in the prevention of several pathological conditions, such as cancer, heart disease, and atherosclerosis [6,7]. Antioxidants function by scavenging radicals, donating electrons and hydrogen, reducing peroxides, and quenching superoxides and singlet oxygen. Because of their different reaction pathways, various methods have been developed in different systems to study free radical-scavenging antioxidant activity. For example, diphenylpicrylhydrazyl (DPPH), 2,2-azobis-(3-ethyl-benzothiazoline-6-sulfonate (ABTS), oxygen radical absorbance capacity (ORAC), and ferric-reducing antioxidant power (FRAP) assays are extensively used as in vitro tests for estimating antioxidant potential [8-10]. However, each assay involves different radicals, demonstrating that single-antioxidant assay may not provide complete information. Therefore, multiple assays have been used to screen the antioxidant capacity of various plant extracts, including fruits and vegetables [11-14].

There is enormous variability in the phenolic compounds and antioxidant activity in different commercial fruits, vegetables, and juices [12,15-17]. Pomegranate and blueberry fruits are considered to be superfoods for their antioxidant activity. Pomegranate, which is popularly consumed as juice, contains ellagitannins, gallotannins, ellagic acid, flavonoids such as anthocyanin and epicatechin, and phenolic acid [16,18]. Blueberries are one of the richest sources of antioxidant phytonutrients, with higher levels of anthocyanins, proanthocyanins, phenolic acid, and flavonols than many other fruits and vegetables. Because of their diverse range of phytonutrients, blueberry and pomegranate have beneficial effects on human health, including anticancer, antidiabetic, anti-neurodegenerative, antiinflammatory, and antiobesity effects $[4,19]$. Potato ranks as one of the major food crops, after rice, wheat, and maize, and breeding new potato cultivars with higher levels of antioxidant compounds is considered a realistic approach to increasing dietary antioxidant intake [20-22]. As a result, highly nutritious potato cultivars with distinctive flesh and skin colors are being developed [20,23]. Potato tubers with red and purple flesh are known to have high phenolic contents [21,22]. Common phenolic acids in potatoes are chlorogenic acid, caffeic acid, cinnamic acid, p-coumaric acid, ferulic acid, and sinapic acid [24]. The predominant phenolic acid in potatoes, chlorogenic acid, constitutes approximately $80 \%$ of the total phenolics [25]. The predominant flavonoid compounds in potato tubers are catechins and epicatechins. In addition to common phenolics, red- and purplefleshed potato tubers contain high amounts of acylated anthocyanins [23]. Red-fleshed potatoes contain acylated glucosides of pelargonidin, whereas purple potatoes also contain acylated glucosides of malvidin, petunidin, peonidin, and delphinidine [26].

Antioxidant activity describes the ability of redox molecules in foods and biological systems to scavenge free radicals, considering the additive and synergistic effects of all antioxidants rather than the effects of single compounds. Thus, in terms of total phenolics (TP), total flavonoids (TF), and total anthocyanins (TA), dietary antioxidants are closely associated with Total Antioxidant Capacity (TAC). However, the TAC consumed by an individual depends on the type and amount of food intake. Therefore, the estimation of TP, TF, and TA and their respective TAC per serving size provides valuable information on

*Corresponding author: Sastry S Jayanty, Department of Horticulture and Landscape Architecture, San Luis Valley Research Center, Colorado State University, 0249 East County, Road 9N Center, CO 81125, USA, E-mail: sastry. jayanty@colostate.edu

Received June 03, 2014; Accepted August 22, 2014; Published August 29, 2014

Citation: Kalita D, Jayanty SS (2014) Comparison of Polyphenol Content and Antioxidant Capacity of Colored Potato Tubers, Pomegranate and Blueberries. J Food Process Technol 5: 358. doi:10.4172/2157-7110.1000358

Copyright: $(2014$ Kalita D, et al. This is an open-access article distributed under the terms of the Creative Commons Attribution License, which permits unrestricted use, distribution, and reproduction in any medium, provided the original author and source are credited. 
potential health benefits [27]. Moreover, the marketing of so-called superfoods is commonly based on their antioxidant potential. In fact, a superior antioxidant activity with health benefits has been claimed for a number of antioxidant foods based on in vitro antioxidant assays. Although various reports are available on polyphenols and the antioxidant capacities of various fruits and vegetables, comparisons of the antioxidant capacity of colored-flesh potato tubers with phenolicrich fruits are scarce.

The main objective of this study was to measure the TP, TF, and TA in red/purple potato, pomegranate juice, and blueberry and to assess their potential antioxidant activities. Three different antioxidant assays, i.e., ORAC, ABTS, and DPPH, were chosen for these measurements, and the relative antioxidant capacity per standard serving size of each food was determined.

\section{Materials and Methods}

\section{Chemicals and materials}

Folin-Ciocalteu Reagent (FCR), sodium carbonate, gallic acid, aluminum trichloride, quercetin hydrate, potassium chloride, sodium acetate, DDPH, ABTS, potassium persulfate, Trolox, AAPH, fluorescein, potassium dihydrogen phosphate, and dipotassium hydrogen phosphate were purchased from Sigma Aldrich Corporation (St. Louis, USA). All other chemicals were of analytical grade.

Concentrated pomegranate juice (100\% pure) was purchased from Dynamic Health Laboratories, Inc ${ }^{\circledast}$. Brooklyn, New York (Lot 688781), and dried blueberries were purchased from Earthy Delight, Dewitt, Michigan (Lot 42561). Potato tubers were collected from the harvest of September 2012 at San Luis Valley Research Center, Center, Colorado. Some of the tubers were pierced twice on each side with a fork and baked at $204^{\circ} \mathrm{C}$ for $1 \mathrm{~h}$ in a commercial oven, as described by Pavek and Kowles [28]. All samples were freeze dried (FreeZone 6, Labconco, Kansas City, MO) with a $0.12-\mathrm{mBar}$ vacuum at $-50^{\circ} \mathrm{C}$. The freeze-dried samples were ground to a fine powder in a grinder and stored in airtight plastic zipper bags at $-80^{\circ} \mathrm{C}$ until analyzed.

\section{Extraction of phenolics}

Phenolics, flavonoids, and anthocyanins were extracted from the food materials stored as mentioned in section 2.1. Five grams of freeze-dried material was weighed into a $50-\mathrm{ml}$ falcon tube, and $50 \mathrm{ml}$ of aqueous $80 \%$ methanol was added. The mixture was homogenized for $5 \mathrm{~min}$ and then incubated overnight in an orbital shaker at 200 $\mathrm{rpm}$ at $25^{\circ} \mathrm{C}$. The homogenates were centrifuged at $10,000 \mathrm{rpm}$ for $10 \mathrm{~min}$, followed by filtration through Whatman (Number 400) filter paper. The residues were re-extracted under the same conditions. The combined filtrates were evaporated under nitrogen gas using a Reacti Vap with a nitrogen gas spray unit (Model 18780, Pierce Chemical Company, Rockford, IL) until the final volume was less than $50 \mathrm{ml}$. The final volume of all the samples was adjusted to $50 \mathrm{ml}$ with methanol and kept at $-80^{\circ} \mathrm{C}$ prior to analysis.

\section{Analysis of total phenolics}

A $20-\mu \mathrm{l}$ aliquot of each extract was mixed with $50 \mu \mathrm{l}$ of distilled water in a 96-well flat-bottom assay plate (Costar 3370, Corning, NY); $50 \mu \mathrm{l}$ of a commercial FCR solution (MP Biomedical, Solon, $\mathrm{OH}$ ) was added and mixed well for $1 \mathrm{~min}$ in a plate reader (Power Wave XS2, BioTek Instruments, Winooski, VT). After $5 \mathrm{~min}, 80 \mu \mathrm{l}$ of 75 gl-1 sodium carbonate solution was added and immediately mixed with a pipette. The contents of the microplate were shaken for $4 \mathrm{~min}$ in the plate reader. The plates were incubated at $25^{\circ} \mathrm{C}$ in the dark, agitating at $150 \mathrm{rpm}$ on an orbital shaker for $2 \mathrm{~h}$. The absorbance of the contents was measured at $760 \mathrm{~nm}$. Gallic acid in methanol was used as the standard, and the total phenolic values were quantified as $\mu \mathrm{g}$ of gallic acid equivalent per gram of dry weight materials using a 5-point calibration curve with an $\mathrm{R}^{2}$ value of 0.989 .

\section{Analysis of total flavonoids}

Equal amounts of $(80 \mu \mathrm{l})$ of the extract and $\mathrm{AlCl} 3$ (20gl-1) ethanolic solution were added to a 96-well flat-bottom microplate on ice. After shaking for $30 \mathrm{sec}$ in a plate reader, the plates were sealed with Parafilm and kept in the dark at $25^{\circ} \mathrm{C}$ for $1 \mathrm{~h}$. The plates were shaken again for 30 $\mathrm{s}$, and the absorbance of the reaction was measured at $415 \mathrm{~nm}$ using the plate reader. Quercetin hydrate in methanol was used as the standard, and the total flavonoids were expressed as $\mu \mathrm{g}$ of quercetin equivalents (QE) per $g$ of freeze-dried materials using a 5-point calibration curve with an $\mathrm{R}^{2}$ value of 0.998 .

\section{Analysis of total anthocyanins}

The total monomeric anthocyanins were determined by the $\mathrm{pH}$ differential method. A $10-\mu \mathrm{l}$ aliquot of the methanol extract of each food material was added separately to $290 \mu \mathrm{l}$ of buffer of potassium chloride ( $\mathrm{pH} 1.0)$ and sodium acetate $(\mathrm{pH} 4.5)$. The absorbance was measured at 515 and $700 \mathrm{~nm}$ for both sets of pH 1.0 and 4.5 solutions using water as a blank. The total anthocyanin content was calculated using the following equation:

Total monomeric anthocyanin $=(\mathrm{A} \times \mathrm{MW} \times \mathrm{DF} \times 1000) / \varepsilon \times 1$

where $\mathrm{A}=(\mathrm{A} 515-\mathrm{A} 700) \mathrm{pH} 1.00-(\mathrm{A} 515-\mathrm{A} 700) \mathrm{pH} 4.5$;

MW $=449.2$ and $\varepsilon=26,900$ are molecular weight and molar absorptivity of cyanidine-3-glucoside, respectively, which was used as the standard; DF is the dilution factor; and $l$ is the path length. The total anthocyanins were reported on the basis of $\mathrm{mg} / \mathrm{g}$ dry weight potato tubers.

\section{Antioxidant activity determinations}

DPPH assay: Aliquots $(20 \mu \mathrm{l})$ of the extract were added to $20 \mu \mathrm{l}$ of distilled water in a 96-well flat-bottom microplate on ice. $200 \mu$ of 120 mg-l DPPH radical solution (using methanol as a solvent) was then added and mixed thoroughly. The absorbance was measured using the plate reader at $515 \mathrm{~nm}$ after keeping the plates in the dark for 30 min on ice. A control with $20 \mu \mathrm{l}$ of methanol (no extract) was also included in each plate. The DPPH radical-scavenging activity was calculated with the following formula:

DPPH radical-scavenging activity $(\%)=[($ Acontrol - Asample/ Acontrol) $] \times 100$

where $\mathrm{A}$ is the absorbance at $515 \mathrm{~nm}$.

ABTS assay: The ABTS assay was performed by adopting the method of Arnao et al. [29]. The stock solutions included an $8 \mathrm{mM}$ ABTS radical solution and a $3 \mathrm{mM}$ potassium persulfate solution. The working solution was prepared by mixing the two stock solutions in equal quantities and allowing them to react for $12 \mathrm{~h}$ at room temperature in the dark. The solution was then diluted by mixing $1 \mathrm{ml}$ ABTS radical solution with $60 \mathrm{ml}$ methanol to obtain an absorbance of approximately 1 unit at $734 \mathrm{~nm}$ using the microplate reader. Sample extract $(150 \mu \mathrm{l})$ was allowed to react with $2850 \mu \mathrm{l}$ of the ABTS solution for $2 \mathrm{~h}$ in the dark. The absorbance was then measured at $734 \mathrm{~nm}$ using the plate reader. The ABTS antioxidant capacities were expressed in $\mu$ mol Trolox equivalents $(\mu \mathrm{mol} \mathrm{TE}) / \mathrm{g}$ 
ORAC assay: Both hydrophilic and lipophilic ORAC assays were carried out using a Cary Eclipse fluorescence spectrophotometer following the method described by Prior et al. [30], with modifications. AAPH was used as a peroxyl generator and was prepared fresh for each run. Fluorescein was used as the substrate. In a quartz cuvette, a 3-ml reaction mixture was prepared by mixing $100 \mathrm{mg} \mathrm{AAPH}, 0.05$ $\mu \mathrm{M}$ fluorescein, and $30 \mu \mathrm{l}$ sample extract in phosphate buffer $(75 \mathrm{mM}$, $\mathrm{pH}$ 7.0). The solution was shaken and equilibrated for $2 \mathrm{~min}$. The fluorescence conditions were as follows: excitation at $485 \mathrm{~nm}$ and emission at $520 \mathrm{~nm}$. The integrate function was used to determine the total area under the curve for each sample. The total antioxidant capacity or ORAC values were expressed as $\mu \mathrm{mol} \mathrm{TE} / \mathrm{g}$ of mass and calculated as follows: Sblank),

ORAC value $(\mu \mathrm{mol} \mathrm{TE} / \mathrm{g})=$ hkc (Ssample - Sblank)/(STrolox -

where $\mathrm{h}$ is the ratio between liters of juice and grams of fruits or vegetables, $\mathrm{k}$ is the dilution factor, and $\mathrm{c}$ is the concentration of Trolox in $\mu \mathrm{mol} / \mathrm{L}$.

For the lipophilic antioxidant activity assay, a dried hexane extract was dissolved in acetone and then diluted with 7\% RMCD (Randomly Methylated Beta Cyclodextrine, www.cyclodex.com) solution (50\% acetone $/ 50 \%$ water). The fluorescein solution and AAPH were added in the same manner as described for the hydrophilic assay.

\section{Statistical analysis}

All experiments were carried out in triplicates and the statistical analysis was performed by one-way analysis of varience (ANOVA) at $\mathrm{p} \leq 0.05$ significance level using XLSTAT 2011 (Addinsoft USA, New York, NY). Error bars in figures are based on a calculated Fischers LSD at $\alpha=0.05$ using the the standard error for the LS means and an approximated T-value of 2. A linear regression analysis was performed using Microsoft Office Excel 2010 to evaluate the correlation coefficient.

\section{Results and Discussion}

\section{Total phenolic, total flavonoid, and total anthocyanin contents}

Phenolics, flavonoids, and anthocyanins are the major group compounds that constitute the phytochemicals of various fruits and vegetables that possess health benefits. Reports are available on profiling of phytonutrients in pomegranate juices, blueberries and purple and red potato tubers [15-26]. Since all antioxidant capacity is the result of all individual antioxidants we were refrain from the profiling antioxidants and measured TP, TF, and TA in pomegranate juice, blueberries, and potato tubers with red and purple flesh. The results are shown in Figure 1 (A-C). The amounts of TP were determined using the Folin-Ciocalteu phenol reagent. Significant variations ( $p$ $\leq 0.05$ ) were observed in the content of TP Figure 1 (A). Among the four different foods, pomegranate had the highest levels of TP. The TP content was in the order pomegranate $>$ blueberries $>$ red-flesh potato tuber $($ CO97226-2R/R) $>$ Purple Majesty potato. The short range of the $\mathrm{TP}(4482$ - $11189 \mu \mathrm{g} \mathrm{GAE} / \mathrm{g})$ value from potato tubers to pomegranate might be an indication of comparable antioxidant activity in potato tubers and blueberry and pomegranate. Previous reports indicated that purple-fleshed potato tubers had total phenolic contents comparable to phenolic-rich fruits and vegetables, such as berries and grapes [31]. Moreover, potato tubers with red flesh had a higher phenolic content than the tubers with purple flesh. As potatoes are consumed after cooking, we tested the total phenolic content after processing using the most popular cooking method, baking, and observed a 45 and $57 \%$ reduction of TP after baking in the purple and red potato tubers, respectively. Similar observations of a reduction in TP in potato tubers after processing by different cooking methods such as boiling, microwaving, and baking have been also been reported [22].

Flavonoids are considered to be one of the most important natural phenolics and possess a broad spectrum of chemical and biological activities, including radical-scavenging properties. The total flavonoids $(\mathrm{TF})$ in pomegranate juice, blueberries, and potato tubers are shown in Figure 1B. Significant variations $(\mathrm{p} \leq 0.05)$ were observed in the content of TF. Pomegranate juice had the highest level of total flavonoids whereas Purple Majesty had the lowest level of total flavonoids. The total flavonoid (TF) content was in the following order: pomegranate $>$ blueberries $>$ red-fleshed potato tuber $($ CO 97226-2R/R) $>$ Purple Majesty. Various reports have described flavonoid profiles in pomegranates and blueberries. Potato tubers with red and purple flesh are also reported to have significant levels of flavonoids [22]. The short range of TF (140- $503 \mu \mathrm{g}$ QE/g )from Purple Majesty to pomegranate found in our study might explain the comparable antioxidant activity of these food materials. A 51 and 53\% reduction of total flavonoids was observed in baked tubers of Purple Majesty and CO97226-2R/R, respectively.

Anthocyanins are reported to have significant antioxidant activity and inhibitory effects on lipid peroxidation. The total anthocyanin (TA) content of pomegranate juice, blueberries, and potato tubers are shown in Figure $1(\mathrm{C})$. Significant variations $(\mathrm{p} \leq 0.05)$ were observed in the content of TA. Pomegranate juice had the highest anthocyanin content, whereas potato tuber CO $97226-2 \mathrm{R} / \mathrm{R}$ had the lowest level of anthocyanins. The total anthocyanin content was in the order pomegranate $>$ blueberries $>$ Purple Majesty $>$ red-fleshed potato tuber (CO 97226-2R/R. Interestingly, Purple Majesty had a similar levels of TA content as blueberry and pomegranate. Moreover, Purple Majesty had higher levels of anthocyanin than the red-fleshed potato tuber (CO97226-2R/R). In the literature very few reports available on anthocyanin content in colored flesh potato tubers. Recently Madiwale et al. [31] have demonstrated that purple flesh potato tubers have an anthocyanin content comparable to that of blueberries. It is interesting to note that Purple Majesty has a considerable amount of total anthocyanins, even after cooking.

\section{Antioxidant activity measured in methanol extract}

DPPH radical-scavenging activity: DPPH (1,1-diphenyl-2picrylhydrazyl) is one of the most reliable, accurate, and frequently employed methods for evaluating antioxidant activity. DPPH is a stable free radical that accepts an electron or hydrogen, with a corresponding change from a violet color to pale yellow in solution, and has been widely used to test the free radical-scavenging ability of various samples. The reduction capability of the DPPH radical was determined by a decrease in absorbance at $517 \mathrm{~nm}$ induced by antioxidants. Figure $2 \mathrm{~A}$ shows the DPPH-scavenging activity of the methanolic extracts of pomegranate juice, blueberry, and potato tubers. Among the four foods, pomegranate juice had the highest DPPH activity, followed by blueberry, red-fleshed potato tuber (CO97226-2R/R) and Purple Majesty with significant variations $(\mathrm{p} \leq 0.05)$.

ABTS scavenging activity: The DPPH assay was useful for determining the free radical-scavenging ability of the extracts; however, the DPPH antioxidant activity depends upon many factors, such as the reaction time (relatively slower rate of reaction), the nature of the phenolic compounds, and the redox potential of the extracts. 

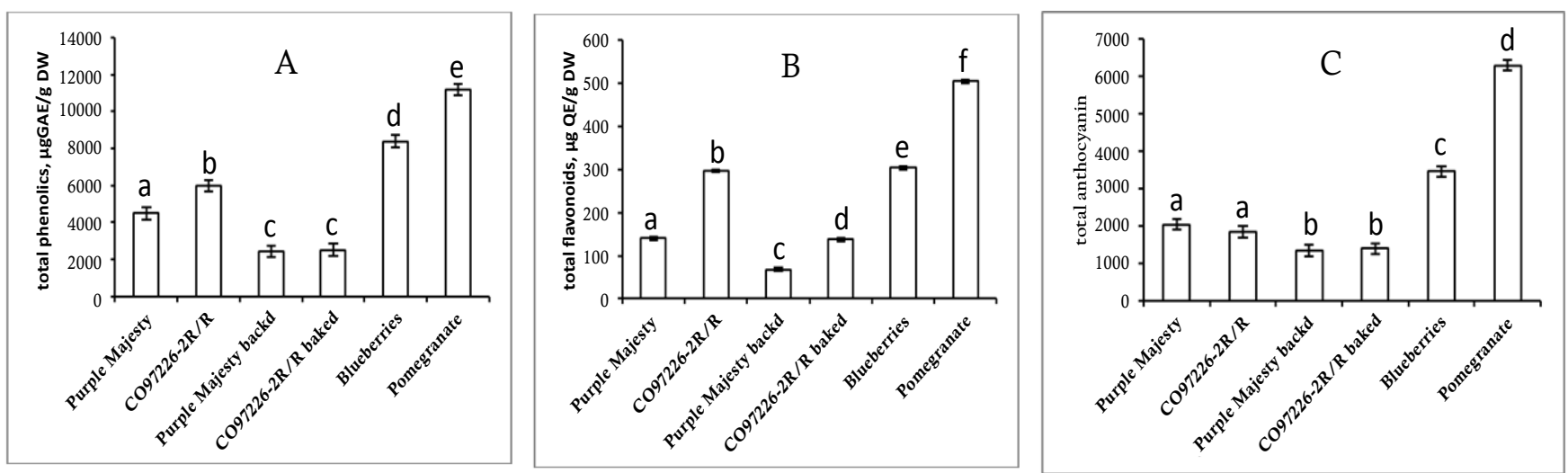

Figure 1: Total phenolics (A), total flavonoids (B), and total anthocyanins $(C)$ in pomegranate, blueberries and potato tubers. Error bars represent the calculated LSD for the least squre means given at $\alpha=0.05$. Significant differences are denoted by different letters, same or shared letters indicate they are not significant each other.

ABTS radicals are more reactive than DPPH radicals, and unlike reactions with DPPH, reactions with the ABTS radical generally occur in less than a millisecond. The assay is based on the reduction of the blue-green ABTS radical by hydrogen-donating antioxidants, and the decay of the ABTS radical is monitored by the absorption at $600 \mathrm{~nm}$. Figure $2 \mathrm{~B}$ shows the ABTS radical cation-scavenging activity of the methanolic extract of pomegranate juice, blueberry, and potato tubers. The pomegranate sample had the highest ABTS value $(258 \mu \mathrm{mol}$ TE / g) than the other extracts. The ABTS antioxidant capacity was in the following order: pomegranate juice $>$ blueberry $>$ red-fleshed potato tuber $($ CO 97226-2R/R) > Purple Majesty with significant variations ( $\mathrm{p}$ $\leq 0.05$ ). The ABTS antioxidant capacity determined by the ABTS assay for the potato tubers was equivalent to that of the pomegranate juice and blueberries.

ORAC antioxidant capacity: The ORAC assay is considered to be more relevant because it utilizes a biologically relevant radical source. The advantage of this assay is that it combines both the inhibition time and the inhibition degree of radical generation, as it takes the oxidation reaction to completion and uses the area under the curve to quantify the antioxidant activity [30]. The total ORAC antioxidant activity of different species is shown in Figure 2C. The ORAC value of pomegranate juice was found to be highest, followed by blueberry and the potato tubers. The total ORAC values of the purple- and red-fleshed potato tubers were similar to blueberry and pomegranate. In addition, the ORAC value for the purple-fleshed tubers occupies a considerable position in the list of antioxidant values of 100 top fruits and vegetables consumed in the USA. Interestingly, cooked potato tubers also have significant antioxidant activity, but with some reduced antioxidant activity, which could be due to the loss of polyphenolic compounds and to the successive formation of other antioxidant compounds such as Maillard reaction products or pyrolysis products (less active). The antioxidant activities using the ABTS radical cation were compared to those derived from the ORAC assay based on the reduction of peroxyl radical by hydrogen-donating antioxidant constituents. The results for the antioxidant activities of pomegranate juice, blueberry, and potato tubers were $258,136,84$, and $109 \mu$ mole TE/g dry weight (ABTS assay) and 706, 496, 301, and $253 \mu \mathrm{mol} \mathrm{TE} / \mathrm{g}$ dry weight (ORAC), respectively.

The lipophilic ORAC antioxidant activity values are shown in Figure $2 \mathrm{D}$, however these values are very low compared to the hydrophilic one. The antioxidant activities obtained from the different assays may reflect a relative difference in the ability of antioxidant compounds in the extracts to quench and reduce aqueous peroxyl, ABTS, and DPPH radicals. The occurrence of different antioxidant activities is the result of the action of several polyphenolic constituents and is not due to single polyphenolic compound.

In general, TP is highly correlated with antioxidant activity. In complex samples such as fruit and vegetable extracts, which may contain many individual polyphenols, some discrepancies between polyphenol content and antioxidant activity could be expected. Nevertheless, in the present work, the data show that the ORAC, ABTS, and DPPH values were strongly correlated with the polyphenol content $(\mathrm{r}=0.9499$, $0.9251,0.8081$ ) (Figures $3 \mathrm{a}-\mathrm{c}$ ). A strong correlation was also obtained between TF and antioxidant activity values for ORAC, ABTS, and $\mathrm{DPPH}$, with $\mathrm{r}=0.8037,0.9138$, and 0.7065 (Figures 3d-f), respectively. Similarly, the anthocyanin content has a strong correlation with the ORAC value with 0.9315 , ABTS value of 0.9645 , and DPPH value of 0.538 (Figure $3 \mathrm{~g}-\mathrm{i}$ ). The high correlation between antioxidant activity and TP, TA, or TF found in our study suggests that it is feasible to use $\mathrm{TP}, \mathrm{TA}$, and TF to screen and compare the antioxidant capacities of potato tubers with other polyphenol-rich fruits and vegetables.

A large body of literature is available regarding the analysis of phenolics and measurements of the antioxidant capacities of various plant extracts, fruits, and vegetables. $\mathrm{Wu}$ et al. [15] estimated TP and the potential antioxidant capacity of over 100 different types of foods, covering fruits, vegetable, nuts, and dried spices consumed in the USA, and a strong positive linear relationship between TP and the antioxidant capacity of fruits and vegetables was obtained. Seeram et al. [16] reported a comparative study of antioxidant potency using the DPPH, ORAC, and FRAP assays for some commonly consumed polyphenol-rich beverages in the USA; the order of antioxidant activity was consistent across the different methods, with the exception of the ORAC method. Borges et al. [17] studied the polyphenolic composition and antioxidant activity of European commercial fruit juices and found that most of the antioxidant activity is due to vitamin $\mathrm{C}$ rather than phenolic compounds. This study demonstrated that ellagitannins, are the major antioxidants in pomegranate juice. Pillai et al. [14] performed a critical analysis of the polyphenolic content and antioxidative capacity of potato tubers using the ORAC and DPPH assays. A very strong antioxidant potential was found in purple-fleshed potato tubers; when compared to other vegetables, potato was found to have the highest antioxidant potential. A similar report is available in which potato tubers with purple flesh had comparable antioxidant capacities to berries and grapes [31]. 

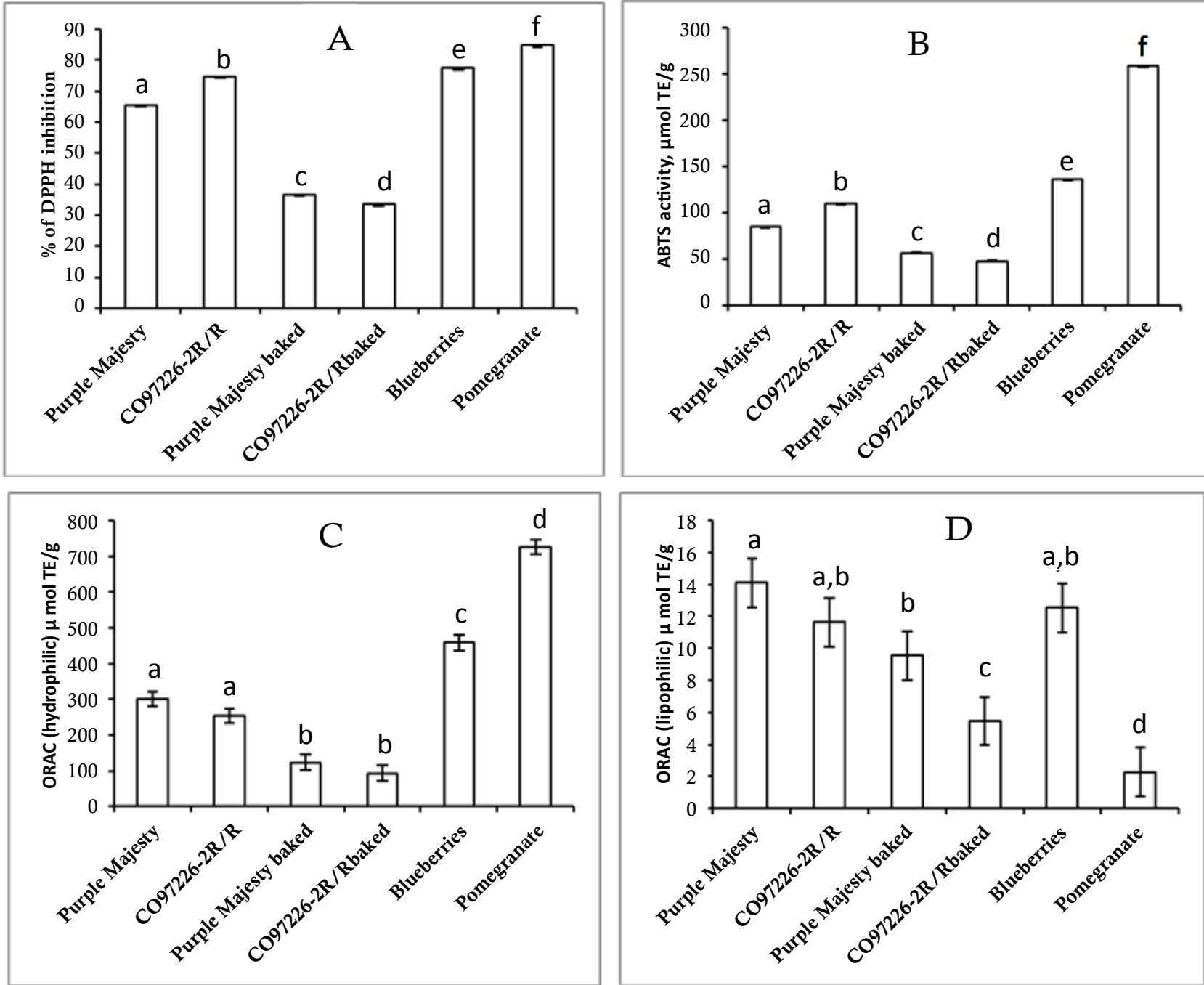

Figure 2: Antioxidant activities of pomegranate, blueberries and potato tubers by (A) DPPH, (B) ABTS, and (C) and (D) ORAC assay (hydrophilic and lipophilic). Error bars represent the calculated LSD for the least squre means given at $\alpha=0.05$. Significant differences are denoted by different letters, same or shared letters indicate they are not significant each other.

There are many differences in the amounts of fruits and juices serving as the recommended daily intakes across countries. For example, the fruit juice requirement is 0.5 cups $(125 \mathrm{ml})$ in Canada, 1 cup $(250 \mathrm{ml})$ in the United States, and 0.6 cups $(150 \mathrm{ml})$ in the United Kingdom. Moreover, the serving sizes of fruits and vegetables also vary across countries. For example, Canada's Food Guide considers $125 \mathrm{ml}$ (0.5 cup) fresh, frozen, or canned vegetable or fruit or 100\% juice, 250 $\mathrm{ml}$ ( 1 cup) leafy raw vegetables or salad, and 1 piece of fruit as one serving. Depending on age, gender, and physical activity level, the US Department of Agriculture (USDA) defines a serving size as from 0.5-2 cups fruits and 1-2.5 vegetables as one serving per meal, distinguishing fruit from vegetables. The United Kingdom recommends that an $80 \mathrm{~g}$ portion correspond to 3 heaping tablespoons of vegetables, 3 heaping tablespoons of beans and pulses, or a dessert bowl of salad. Therefore, to make an overall evaluation of the total antioxidant capacity consumed, serving sizes as well as concentrations must be considered. We determined the total antioxidant capacity of pomegranate, blueberry, and purple/red potatoes per serving size. The standard serving sizes of concentrated pomegranate juice (100\%), dried blueberries, and potato tubers are $30 \mathrm{ml}, 40 \mathrm{~g}$, and $173 \mathrm{~g}$, respectively, in the USA. The total antioxidant activity measured by the ORAC, ABTS, and DPPH assay are shown in Table 1. Under this standard serving condition, the antioxidant activity of red/purple flesh potato tubers is comparable to those of pomegranate juice and blueberries.

Significantly, there are large differences in the price of polyphenolrich blueberries and potato tubers. The prices of one pound of dried blueberries, $8 \mathrm{oz}$ of pomegranate, and one pound of purple potatoes are $\$ 10.95$ (www.earthy.com), \$9.00 (www.walmart.com), and \$1.99 (Whole Foods, Fort Collins, CO), respectively, and \$ 0.97, 1.14, and 0.75 per serving, respectively. Therefore, for beneficial and economic alternatives for improving health in poor communities, where access to meat or the high cost of fruits such as blueberries and pomegranate cause them to be limited, relatively cheaper red- and purple-fleshed potatoes can be considered a good choice. 
Citation: Kalita D, Jayanty SS (2014) Comparison of Polyphenol Content and Antioxidant Capacity of Colored Potato Tubers, Pomegranate and Blueberries. J Food Process Technol 5: 358. doi:10.4172/2157-7110.1000358

Page 6 of 7
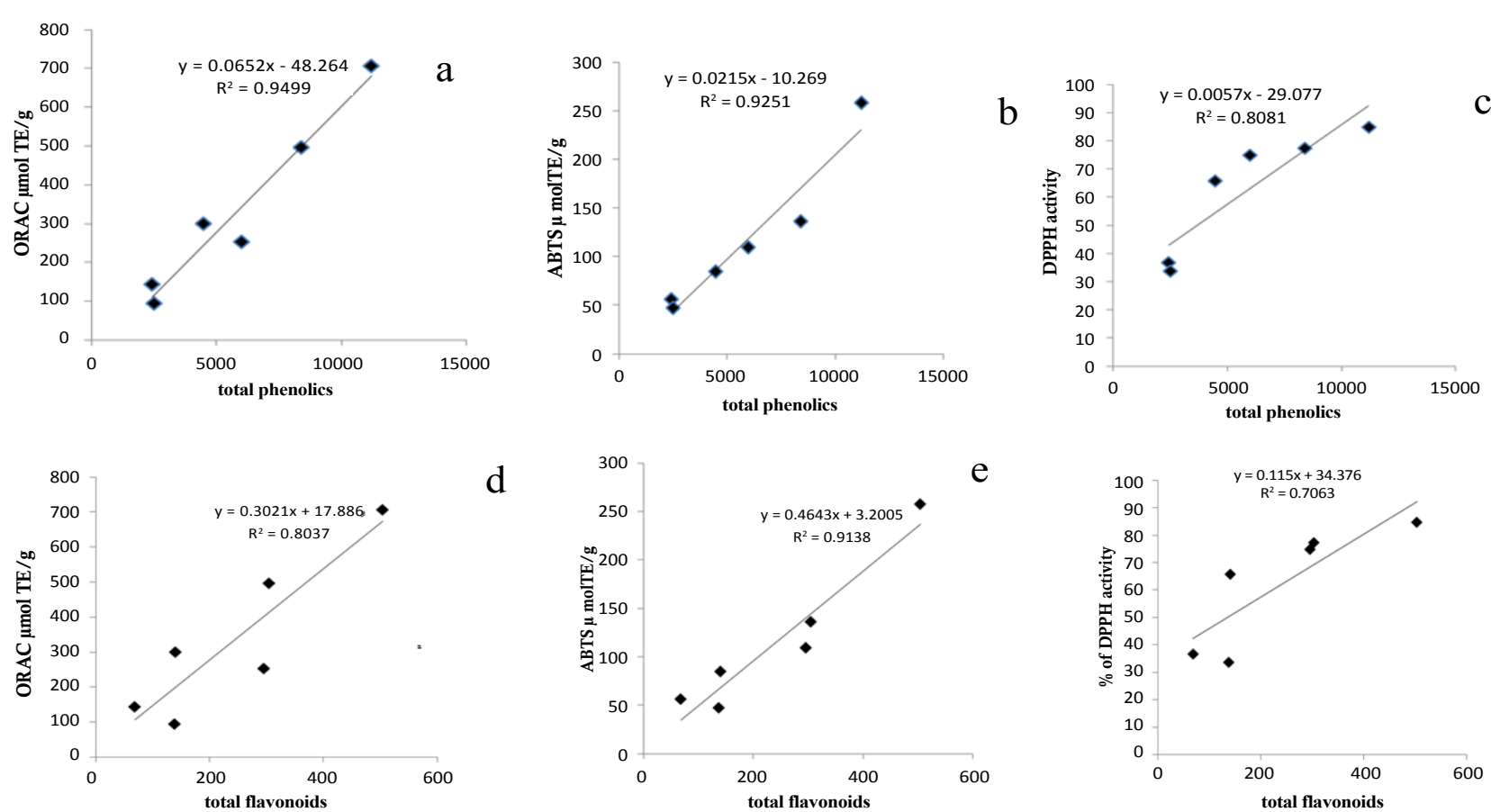

d
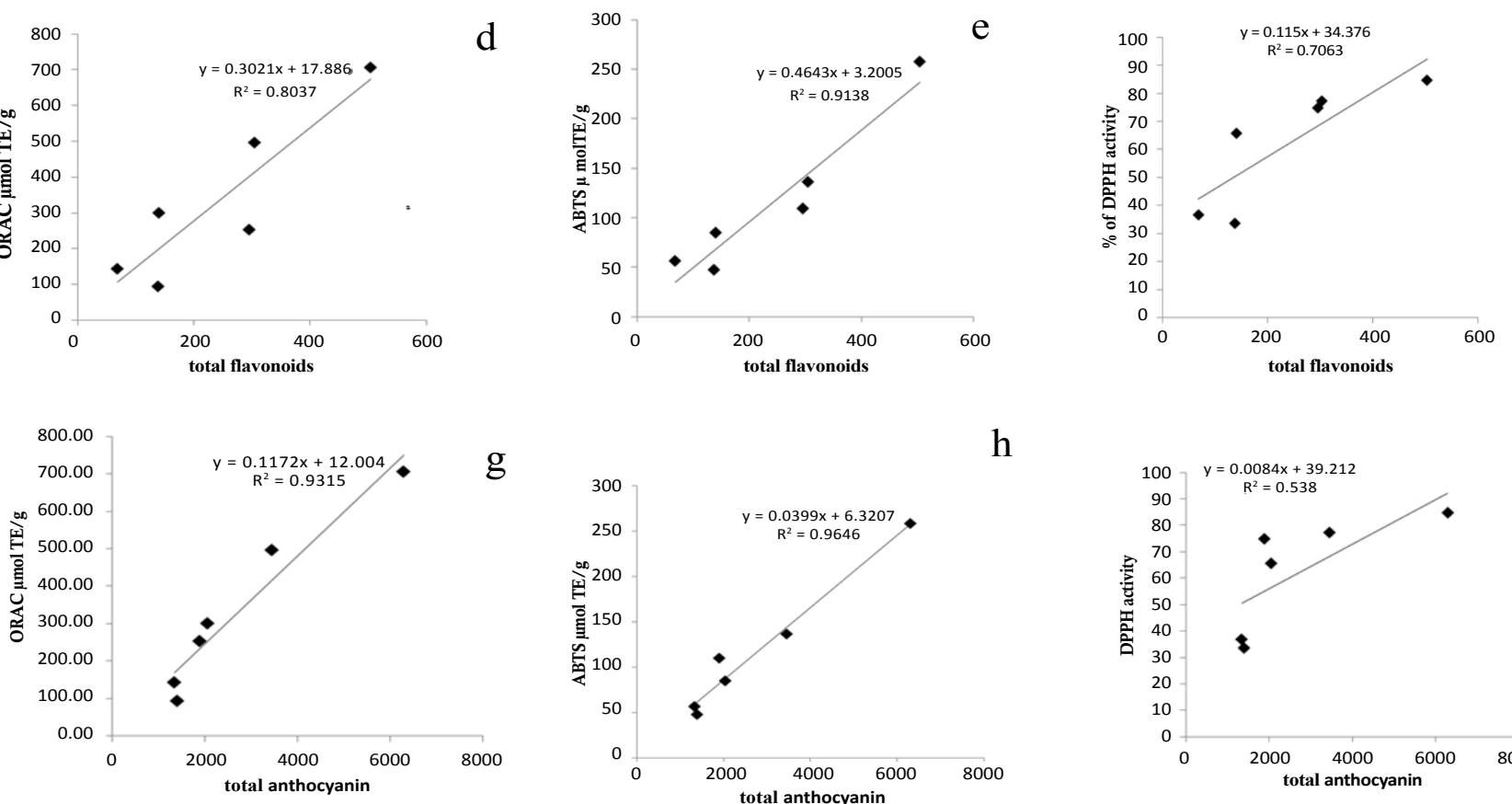

$\mathrm{g}$

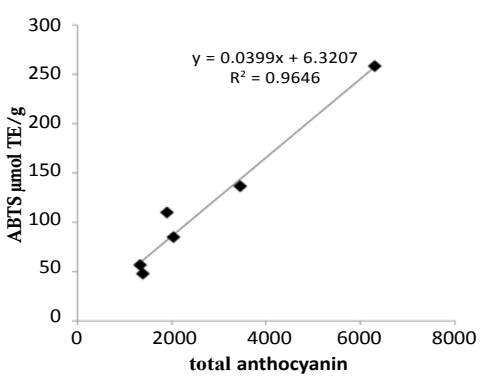

h

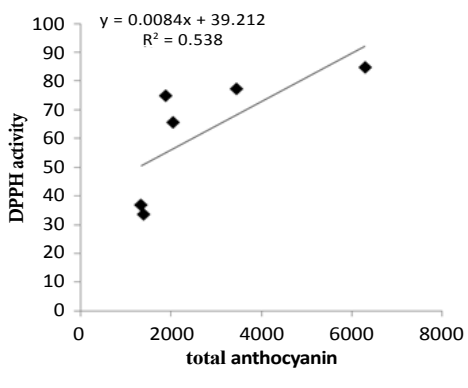

Figure 3. Relationship of total phenolics (a-c), total flavonoids (d-f), and total anthocyanins ( $g-i)$ with ORAC, ABTS, and DPPH assay.

\begin{tabular}{|l|l|l|l|}
\hline Food & Serving Size & \multicolumn{2}{|l|}{ TAC/serving $(\boldsymbol{\mu}$ mol of TE) } \\
\hline & & ORAC & ABTS \\
\hline Pomegranate & $30 \mathrm{ml}$ & 21270 & 7743 \\
\hline Blueberries & $40 \mathrm{~g}$ & 19345 & 5170 \\
\hline & & & \\
\hline Purple Majesty & $173 \mathrm{~g}$ & 13886 & 3946 \\
\hline & & & \\
\hline CO97226-2R/R & $173 \mathrm{~g}$ & 5066 & 3559 \\
\hline
\end{tabular}

Table 1: Total Antioxidant Capacity (TAC) of pomegranate, blueberries, and potato tubers

\section{Acknowledgements}

We thank Dr. Renee Beeton, Faculty of Department of Chemistry, Adams State University for helping us with the use of the fluorescence spectrophotometer and other equipment. Elita Castleberry and Colter Caroll are acknowledged for valuable help in collecting and storing the freeze-dried materials. David G. Holm is acknowledged for providing the potato cultivars and advanced selections.

\section{References}

1. Manach C, Scalbert A, Morand C, Rémésy C, Jiménez L (2004) Polyphenols: food sources and bioavailability. Am J Clin Nutr 79: 727-747.

2. Scalbert A, Andres-Lacueva C, Arita M, Kroon P, Manach C, et al. (2011) Databases on food phytochemicals and their health-promoting effects. J Agric Food Chem 59: 4331-4348.

3. Wang H, Nair MG, Strasburg GM, Chang YC, Booren AM, et al. (1999) Antioxidant and antiinflammatory activities of anthocyanins and their aglycon, cyanidin, from tart cherries. J Nat Prod 62: 294-296.

4. Duthie GG, Duthie SJ, Kyle JA (2000) Plant polyphenols in cancer and heart disease: implications as nutritional antioxidants. Nutr Res Rev 13: 79-106.

5. Lila MA (2004) Anthocyanins and Human Health: An In Vitro Investigative Approach. J Biomed Biotechnol 2004: 306-313.

6. Ames BN, Shigenaga MK, Hagen TM (1993) Oxidants, antioxidants, and the degenerative diseases of aging. Proc Natl Acad Sci U S A 90: 7915-7922.

7. Shi HL, Noguchi N, Niki E (2001) Introducing natural antioxidants. Antioxidants in food: practical applications. Cambridge: Wood head Publishing Ltd. and CRC Press.

8. Huang D, Ou B, Prior RL (2005) The chemistry behind antioxidant capacity assays. J Agric Food Chem 53: 1841-1856. 
Citation: Kalita D, Jayanty SS (2014) Comparison of Polyphenol Content and Antioxidant Capacity of Colored Potato Tubers, Pomegranate and Blueberries. J Food Process Technol 5: 358. doi:10.4172/2157-7110.1000358

9. Cíž M, Cížová H, Denev P, Kratchanova M, Slavov A, et al. (2010) Different methods for control and comparison of the antioxidant properties of vegetables. Food Control 21: 518-523.

10. Müller L, Fröhlich K, Böhm V (2011) Comparative antioxidant activities of carotenoids measured by ferric reducing antioxidant power (FRAP, ABTS bleaching assay (aTEAC), DPPH assay and peroxy radical scavenging assay. Food Chemistry 129: 139-148.

11. Thaipong K, Boonprakob U, Crosby K, Cisneros-Zevallos L, Byrne DH (2006) Comparison of ABTS, DPPH, FRAP, and ORAC assays for estimating antioxidant activity from guava fruit extracts. Journal of Food Composition and Analysis 19: 669-675

12. Lim YY, Lim TT, Tee JJ (2007) Antioxidant properties of several tropical fruits: A comparative study. Food Chemistry 103: 1003-1008.

13. Teow CC, Truong Ven D, McFeeters RF, Thompson RL, Pecota KV, et al. (2007) Antioxidant activities, phenolic and beta-carotene contents of sweet potato genotypes with varying flesh colors. Food Chemistry 103: 829-838.

14. Pillai SS, Navarre Du RA, Bamberg J (2013) Analysis of polyphenols, anthocyanins, and carotenoids in tubers from Solanum tuberosum group phureja, stenotomum and andigena. American Journal of Potato research 90 $440-450$

15. Wu X, Beecher GR, Holden JM, Haytowitz DB, Gebhardt SE, et al. (2004) Lipophilic and hydrophilic antioxidant capacities of common foods in the United States. J Agric Food Chem 52: 4026-4037.

16. Seeram NP, Henning SM, Zhang Y, Suchard M, Li Z, et al. (2006) Pomegranate juice ellagitannin metabolites are present in human plasma and some persist in urine for up to 48 hours. J Nutr 136: 2481-2485

17. Borges G, Mullen W, Crozier A (2010) Comparison of the polyphenolic composition and antioxidant activity of European commercial fruit juices. Food Funct 1: 73-83.

18. Gil MI, Tomás-Barberán FA, Hess-Pierce B, Holcroft DM, Kader AA (2000) Antioxidant activity of pomegranate juice and its relationship with phenolic composition and processing. J Agric Food Chem 48: 4581-4589.

19. Aviram M, Dornfeld L, Rosenblat M, Volkova N, Kaplan M, et al. (2000) Pomegranate juice consumption reduces oxidative stress, atherogenic modifications to LDL, and platelet aggregation: studies in humans and in atherosclerotic apolipoprotein E-deficient mice. American Journal of Clinical Nutrition 71: 1062-1076.
20. Stushnoff C, Holm DG, Thompson MD, Jiang W, Thompson HJ, et al. (2008) Antioxidant properties of cultivars and selections from the Colorado potato breeding program. American Journal of Potato Research 85: 267-276.

21. Navarre Du RA, Pillai SS, Shakya R, Holden MJ (2011) HPLC profiling of phenolics in diverse potato genotypes, Food chemistry 127: 34-41.

22. Perla V, Holm DG, Jayanty SS (2012) Effects of cooking methods on polyphenols, pigments and antioxidant activity in potato tubers, LWT-Food Science and Technology 45: 161-171.

23. Lachman J, Hamouz K (2005) Red and purple colored potatoes as a significan antioxidant source of human nutrition - a review. Plant, Soil and Environment 51: 477-482.

24. Friedman M (1997) Chemistry, biochemistry, and dietary role of potato polyphenols. A review. Journal of Agriculture and Food Chemistry 45: 15231540

25. Brown CR (2005) Antioxidants in potato. American Journal of Potato Research 82: 163-172

26. Lachman J, Hamouz K, Sulc M, Orsak M, Pivec V, et al., (2009) Cultivar differences of total anthocyanins and anthocyanidins in red and purple fleshed potatoes and their relation to antioxidant activity. Food Chemistry 114: 836-843.

27. Wang H, Cao GH, Prior RL (1996) Total antioxidant capacity of fruits. Journa of Agricultural and Food Chemistry 44: 701 - 705.

28. Pavek MJ, Knowles NR (2009) Potato cultivar yield and postharvest quality evaluations, Washington State University, Potato Research Group, Pullman, WA, USA

29. Arnao MB, Cano A, Acosta M (2001) The hydrophilic and lipophilic contribution to total antioxidant activity. Food Chemistry $73: 239-244$

30. Prior RL1, Hoang H, Gu L, Wu X, Bacchiocca M, et al. (2003) Assays fo hydrophilic and lipophilic antioxidant capacity (oxygen radical absorbance capacity (ORAC(FL))) of plasma and other biological and food samples. J Agric Food Chem 51: 3273-3279.

31. Madiwale GP, Reddivari L, Stone M, Holm DG, Vanamala J (2012) Combined effects of storage and processing on the bioactive compounds and proapoptotic properties of color-fleshed potatoes in human colon cancer cells Journal of agricultural and food chemistry 60: 11088-11096. 